\title{
Apodization Optimization of FBG Strain Sensor for Quasi-Distributed Sensing Measurement Applications
}

\author{
Fahd Chaoui, ${ }^{1}$ Otman Aghzout, ${ }^{2}$ Mounia Chakkour, ${ }^{1}$ and Mounir El Yakhloufi ${ }^{3}$ \\ ${ }^{1}$ Department of Physics, Faculty of Sciences, UAE, Tetouan, Morocco \\ ${ }^{2}$ Electronics \& Microwaves Group, Telecommunication Department, ENSA, UAE, Tetouan, Morocco \\ ${ }^{3}$ Condensed Matter Physics Group, Faculty of Sciences, UAE, Tetouan, Morocco
}

Correspondence should be addressed to Fahd Chaoui; fahdchaoui@outlook.com

Received 14 September 2016; Accepted 13 November 2016

Academic Editor: Gerard Ghibaudo

Copyright (C) 2016 Fahd Chaoui et al. This is an open access article distributed under the Creative Commons Attribution License, which permits unrestricted use, distribution, and reproduction in any medium, provided the original work is properly cited.

\begin{abstract}
A novel optimized apodization of Fiber Bragg Grating Sensor (FBGS) for quasi-distributed strain sensing applications is developed and introduced in this paper. The main objective of the proposed optimization is to obtain a reflectivity level higher than $90 \%$ and a side lobe level around $-40 \mathrm{~dB}$, which is suitable for use in quasi-distributed strain sensing application. For this purpose, different design parameters as apodization profile, grating length, and refractive index have been investigated to enhance and optimize the FBGS design. The performance of the proposed apodization has then been compared in terms of reflectivity, side lobe level (SLL), and full width at half maximum (FWHM) with apodization profiles proposed by other authors. The optimized sensor is integrated on quasi-distributed sensing system of 8 sensors demonstrating high reliability. Wide strain sensitivity range for each channel has also been achieved in the quasi-distributed system. Results prove the efficiency of the proposed optimization which can be further implemented for any quasi-distributed sensing application.
\end{abstract}

\section{Introduction}

Over the last 20 years, optical sensors technology has been a major user of the enormous growth of the technology associated with optoelectronics and optical fiber communication industry. Many of the components associated with these industries were often developed for optical fiber sensing applications. The inherent advantages of optical fiber sensors which include high sensitivity, low power, wide bandwidth, and insensitivity to electromagnetic radiations offer wide usage in places where other sensors are unable to function properly [1]. Fiber Bragg Grating Sensor (FBGS) in turn is proving to be one of the most important recent developments in optical sensors technology. It basically constitutes generalized distributed reflector whose reflection spectrum and dispersion characteristics are wavelength-dependent and can be accurately adjusted by a proper design to measure strain and temperature [2]. The important feature of FBGS is that the sensed information is translated into a wavelength shift, giving reproducible measurements in spite of optical losses and intensity fluctuations [3]. However, FBGS with constant refractive index modulation amplitude show reflection spectrum with large side lobes [4], as well as highly nonlinear dispersion characteristics, which makes them unsuitable for high-performance applications. For this reason, apodized FBGS has been widely developed and applied in optical fiber technology since apodized grating has superior filtering performance and high side lobe suppression. The reflection spectrum of an apodized and periodic grating follows closely the Fourier transform of the applied apodization profile. As a result, smooth and tight apodization results in enhanced side lobe suppression and superior grating performance [5]. In this work, we investigate thoroughly the FBGS apodization performance based on reflectivity level, full width at half maximum (FWHM), and side lobe level (SLL) across different values of grating length, refractive index modulation depth, and different apodization profiles in order to get optimized reflection spectrum with high reflectivity and efficient side lobe suppression. The main objective of this procedure is to find out the optimized 


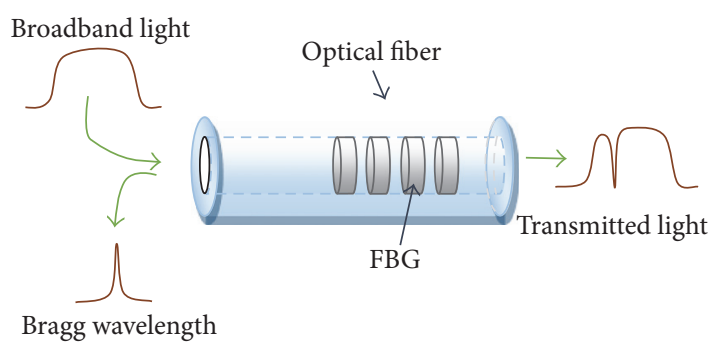

FIGURE 1: Fiber Bragg grating.

setting of the FBGS for use in strain measurement of quasidistributed sensing systems with 8 sensors. The rest of the paper is organized as follows. In Section 2 we briefly describe the general theoretical model used for the calculation of the grating response. The main apodization profiles considered in this paper are also presented in this section. In Section 3, we compare the effect of the apodization profile on reflectivity, FWHM, and side lobe level of the sensor grating. The comparison is further quantified to find out the most suitable setting of the FBG sensor to be used in strain measurement. Once the optimized sensor is designed and simulated, it is implemented in quasi-distributed sensing system of 8 sensors to test its reliability in Section 4. The maximum allowable strain range is determined and can be measured using one channel. The conclusions are given in Section 5.

\section{FBGS Formulation}

In order to develop the new method of apodization based on the FBG technology, firstly we present a brief overview of Bragg grating reflectivity and how the process of apodization helps to get better reflection spectrum. Then, we compare the main apodization profiles discussed in the literature $[3,4]$ with our proposed method to demonstrate the efficiency of our proposed apodization.

FBG is a wavelength selective mirror as can be shown in Figure 1. It reflects the propagated light in fiber according to Bragg wavelength which is given as follows:

$$
\lambda_{B}=2 n_{\mathrm{eff}} \Lambda,
$$

where $n_{\text {eff }}$ is the effective refractive index of the fiber core and $\Lambda$ is the grating period [6].

The refractive index along the grating length varies periodically in the form:

$$
\begin{aligned}
n_{\mathrm{eff}}(z)= & n_{0}+f(z) \Delta n_{a c}(z) \cos \left(\left(\frac{2 \pi}{\Lambda}\right) z+\theta(z)\right) \\
& +n_{2} E^{2}(z),
\end{aligned}
$$

where $z$ is the position, $n_{0}$ is the refractive index prior to grating inscription, $\Delta n_{a c}$ is the refractive index modulation amplitude, $\Delta n_{d c}$ is the average change in refractive index, $f(z)$ is the apodization function, $\theta(z)=(2 \pi / \Lambda) C z^{2}$ is the chirp function, where $C$ is the chirp parameter, $E(z)$ is the electric field, and $n_{2}$ is the nonlinear Kerr coefficient $[2,7]$.
Coupled Mode Theory (CMT) describes the behavior of Bragg grating, where the counter-propagating fields inside the grating structure are related by coupled differential equations [7]. The coupled mode equations that describe the complex amplitudes $A(z)$ and $B(z)$ are given as follows:

$$
\begin{aligned}
& \frac{d A(z)}{d z}=i k B(z) e^{-i(2 \Delta \beta) z}, \\
& \frac{d B(z)}{d z}=-i k^{*} A(z) e^{i(2 \Delta \beta) z},
\end{aligned}
$$

where $\beta$ is the wave propagation constant, $\Delta \beta=\beta-\beta_{0}$ is the differential propagating constant $\left(\beta=2 \pi n_{0} / \lambda ; \beta_{0}=\pi / \Lambda\right)$, and $k$ is the coupling coefficient. For uniform gratings, $k$ is a constant and it is related to the index modulation depth.

Using CMT, the reflectivity of the FBG with constant modulation amplitude and period is given by

$$
R(l, k)=\frac{k^{2} \sinh ^{2}(s l)}{\left(\Delta \beta^{2} \sinh ^{2}(s l)+s^{2} \cosh ^{2}(s l)\right)} .
$$

In this work, we propose to use a new apodization profile as it provides smooth reflection spectrum with high reflectivity and superior filtering performance:

$$
f(z)=0.55-0.45 \cos \left(\frac{2 \pi z}{L}\right) .
$$

The main apodization profiles discussed in literature are given as follows:

(i) Gaussian [3]:

$$
f(z)=\exp \left(-4 \cdot \ln (2) \cdot\left(\frac{2(z-(L / 2))}{L}\right)^{2}\right)
$$

(ii) Nuttall [4]:

$$
\begin{aligned}
f(z)= & a_{0}-a_{1} \cos \left(2 \pi\left(\frac{z}{L}\right)\right)+a_{2} \cos \left(4 \pi\left(\frac{z}{L}\right)\right) \\
& -a_{3} \cos \left(6 \pi\left(\frac{z}{L}\right)\right)
\end{aligned}
$$

$a_{0}=0.3635819, a_{1}=0.4891775, a_{2}=0.1365995$, and $a_{3}=$ 0.0106411 are Nuttall coefficients, $L$ is the grating length, and $z$ is the coordinate of light propagation along the length of FBG.

Figure 2 shows the reflection spectrum of FBG using the different functions of apodization compared to our proposed profile. It can be clearly seen from Figure 2 that uniform grating (without apodization) has high side lobe level compared to apodized gratings. Besides, it can also be observed that Gaussian and Nuttall gratings have less reflectivity than the proposed profile due to the strong apodization process. Strong apodization decreases reflectivity level remarkably which is clearly the case in Nuttall apodization. Figure 3 shows the geometrical shape of the refractive index apodization profiles for Uniform, Gaussian, Nuttall, and the proposed profile, respectively. It can be seen that Uniform has the largest geometrical shape among the apodization profiles while Nuttall has the smallest shape, which affects clearly their reflectivity as previously shown in Figure 2. 

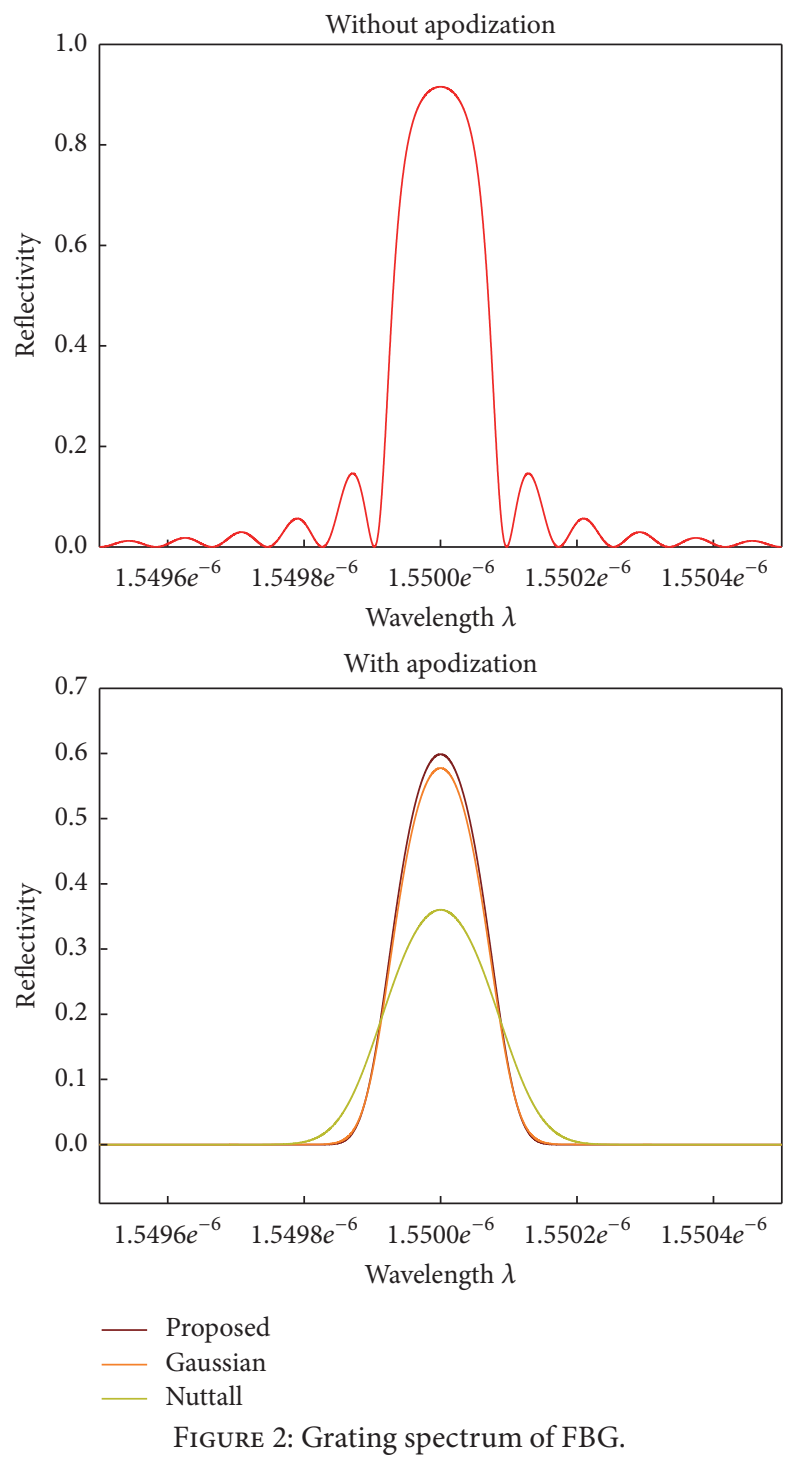

\section{FBGS Optimization}

In this section, the comparison between our proposed profile and the apodization proposed by other authors is further quantified based on different design parameters to prove the efficiency of the proposed profile and to find out the most appropriate setting for strain measurement.

The simulations are performed by assuming that the FBG is inscribed into standard telecommunication single mode optical fiber with $2 \mu \mathrm{m}$ core radius. Bragg wavelength is set to $1550 \mathrm{~nm} . \Delta n_{a c}$ varies from $10^{4}$ to $5 \times 10^{4}$ while $L$ is maintained at $10 \mathrm{~mm}$. Then, the simulations are repeated with varying $L$ from $4 \mathrm{~mm}$ to $20 \mathrm{~mm}$ while $\Delta n_{a c}$ is maintained at $2 \times 10^{4}$. These values are chosen based on previous research papers $[4,8]$. The FBG design setting with high reflectivity, tight FWHM, and high side lobe suppression is then analyzed under strain loads in order to get optimized wavelength shift.

3.1. FBGS Evaluation Based on Refractive Index. Figure 4 illustrates FWHM versus refractive index for the different apodization functions for fixed value of grating length $(L=$
$10 \mathrm{~mm}$ ). In order to determine the acceptable range of $\Delta n_{a c}$, FWHM should be between $0.1 \mathrm{~nm}$ and $0.2 \mathrm{~nm}[4,7]$. Thus, the perfect values of $\Delta n_{a c}$ would be around $2 \times 10^{-4}$.

The reflectivity and side lobe level (SLL) as functions of $\Delta n_{a c}$ while maintaining $L$ at $10 \mathrm{~mm}$ for the different apodization profiles are depicted in Figures 5 and 6, respectively. It can be observed from Figure 5 that our proposed profile provides the highest value compared to Gaussian [3] and Nuttall [4] around $\Delta n_{a c}=2 \times 10^{-4}$, where FWHM is in acceptable range according to Figure 4 .

It can be observed from Figure 6 that our proposed profile offers less side lobe level than Gaussian apodization, whereas Nuttall provides the lowest SLL due to its strong process of apodization, which affects remarkably its reflectivity level as shown in Figure 5.

3.2. FBGS Evaluation Based on Grating Length. Figure 7 shows FWHM versus grating length $L$ for fixed value of refractive index modulation amplitude $\left(\Delta n_{a c}=2 \times 10^{-4}\right)$. In order to determine the acceptable range of $L$, FWHM should be between $0.1 \mathrm{~nm}$ and $0.2 \mathrm{~nm}[4,7]$. Thus, the perfect values of $L$ would be between $10 \mathrm{~mm}$ and $20 \mathrm{~mm}$.

The reflectivity and SLL as function of $L$ while maintaining $\Delta n_{a c}$ at $2 \times 10^{-4}$ for the different apodization profiles are shown in Figures 8 and 9, respectively.

It is shown from Figure 8 that the proposed profile provides clearly the highest value between all the apodized gratings at $n_{a c}=2 \times 10^{-4}$ in the grating length range determined by FWHM. Nuttall profile leads however to strong apodization which results in low reflectivity levels. It can also be noticed from Figure 9 that SLL of the proposed profile is around $-40 \mathrm{~dB}$ which is considered to be very good level. Nuttall profile provides the lowest SLL values at the expense of reflectivity level. It has also been concluded from previous results illustrated in the analysis that the most proper grating length for the optimized apodized FBG sensor equals $20 \mathrm{~mm}$ and 0.0002 is the most convenient refractive index modulation amplitude.

3.3. Comparison Summary. To choose the optimum reflection spectrum of the FBG sensor, performance comparison was carried out based on three thresholds: high reflectivity with more than $90 \%$, narrow FWHM with less than $0.2 \mathrm{~nm}$, and low SLL around $-40 \mathrm{~dB}$. The previous thresholds are suggested with the aid of several sources, vendors, and other literature reviews $[4,7]$. The comparison results show that the proposed profile results in an overall superior performance compared with Gaussian [3] and Nuttall [4] since it provides very good reflectivity and very low SLL at the acceptable range of FWHM. Nuttall profile offers an excellent side lobe suppression, but it wastes an important amount of reflectivity power, which is not convenient in high-performance application, that is why it should only be used when high reflectivity level is not required.

\section{Optimized FBG Strain Sensitivity}

In this section, the optimized FBGS with $L=20 \mathrm{~mm}$ and $d n=0.0002$ is used for strain measurement to test 

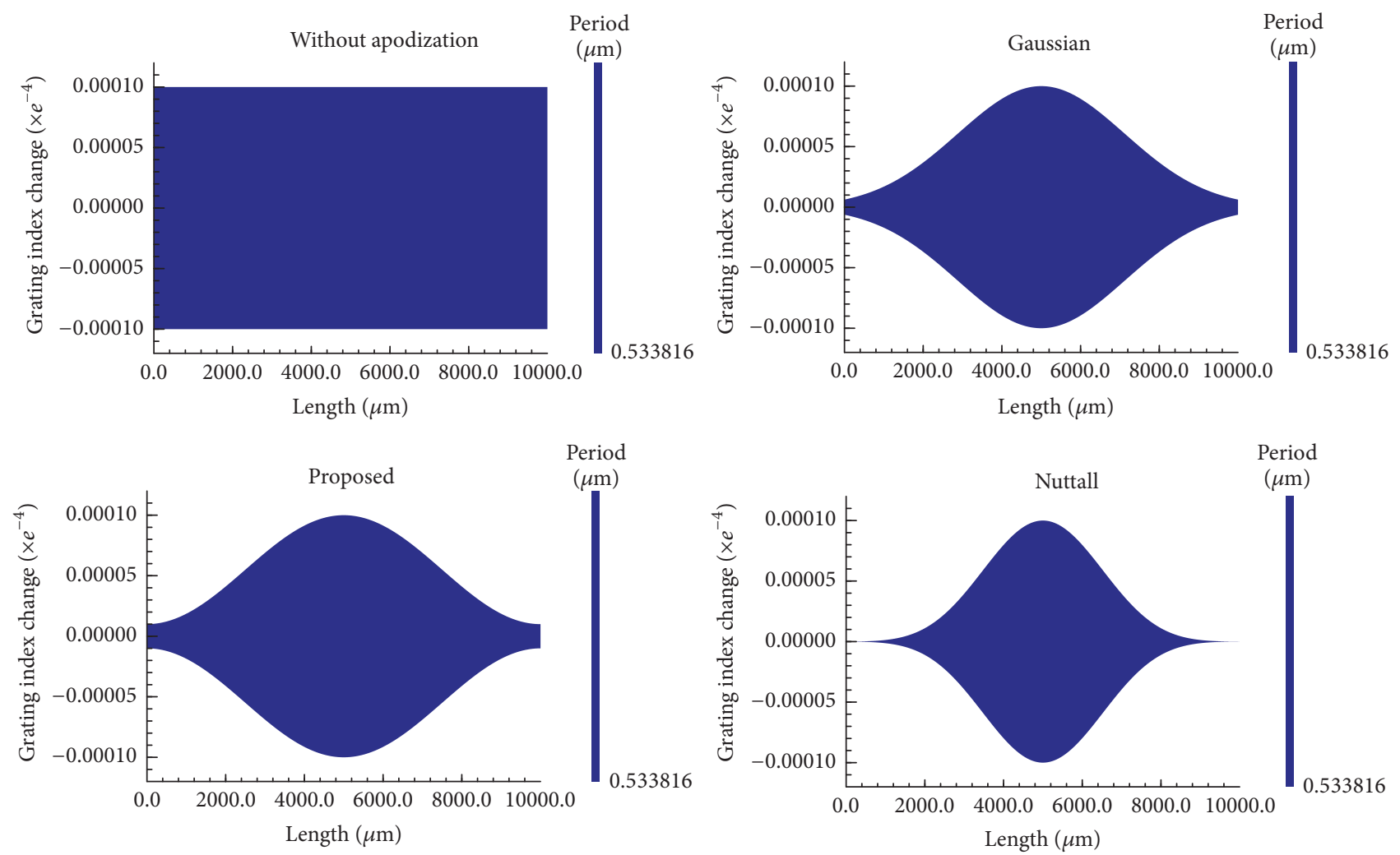

FIgURE 3: Geometrical shape of the apodization profiles.

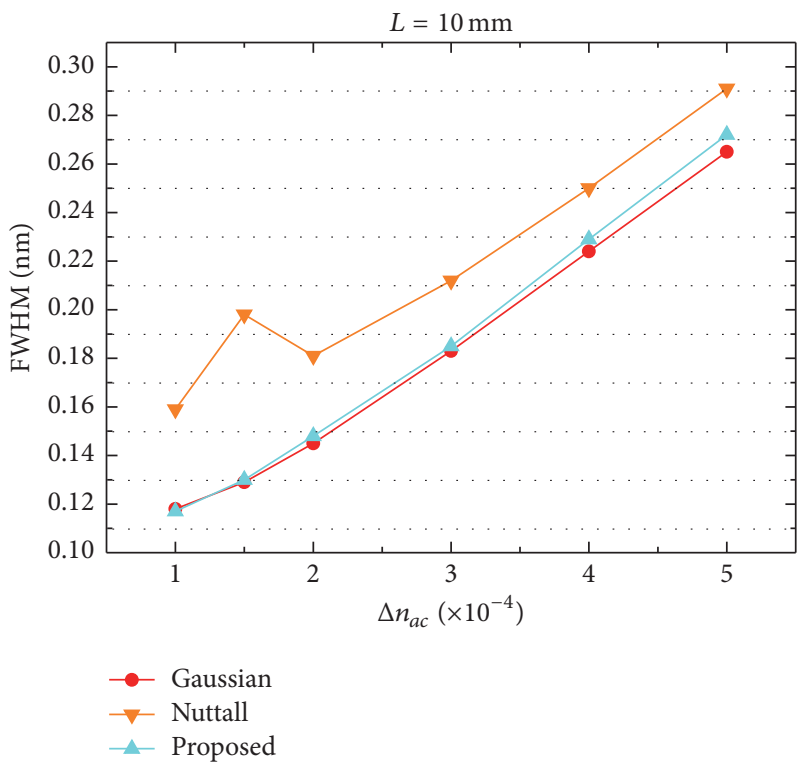

FIGURE 4: FWHM versus refractive index.

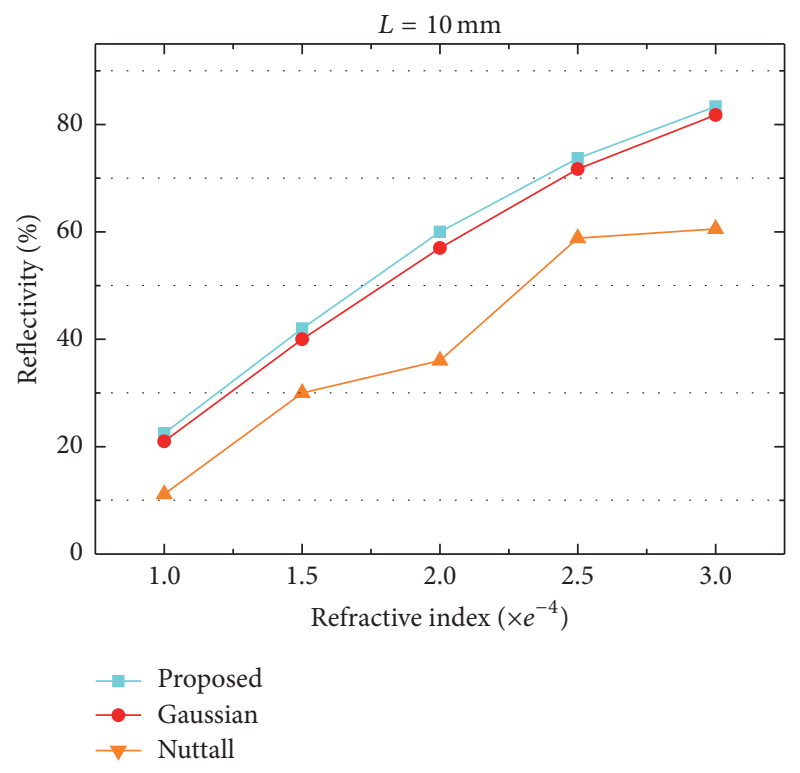

FIgURE 5: Reflectivity versus refractive index. its performance in detection of the small wavelength shift affected by the applied strain loads. Strain in any material is the fractional increase in its physical length when stressed. In linear form, strain $\varepsilon$ is equal to the ratio of the applied stress $\sigma$ divided by Young's modulus of the material $E$ :

$$
\varepsilon=\frac{\sigma}{E}
$$




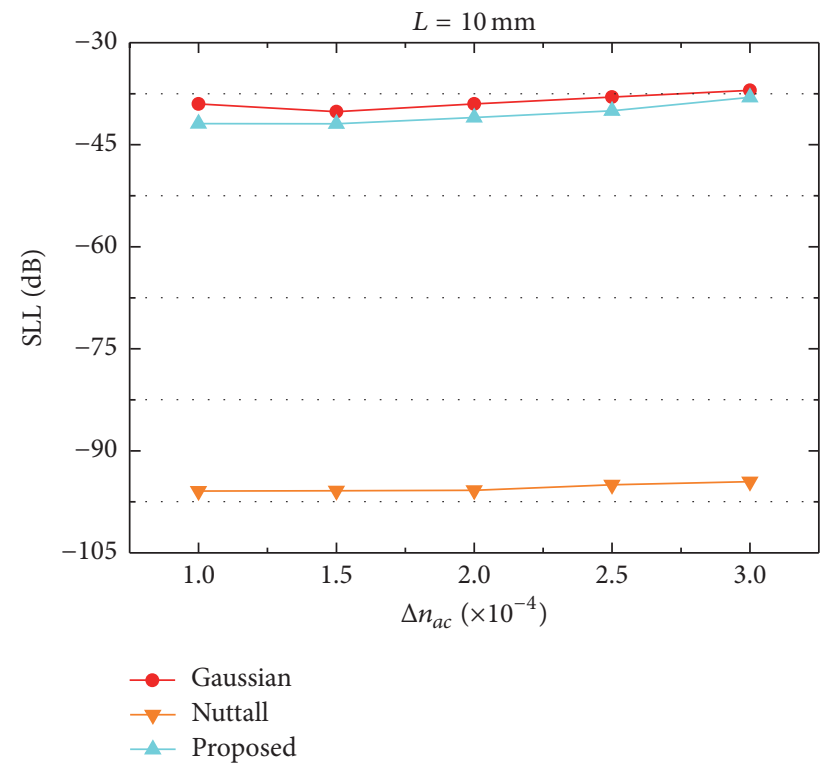

FIGURE 6: Side lobe level versus refractive index.

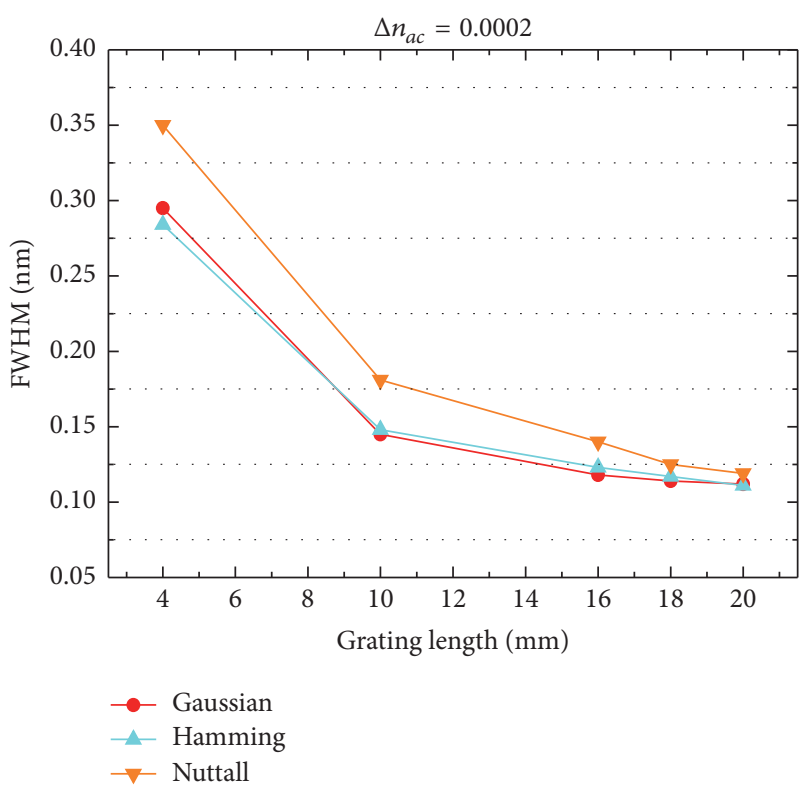

FIGURE 7: FWHM versus grating length.

The strain variation in the Bragg grating portion is determined by the wavelength shift from the sensor reflectivity peak as can be shown in Figure 10. For measuring strain it is necessary to introduce a mechanism that relates the change of strains to the change of refractive index and grating period which can be expressed by

$$
\begin{aligned}
n_{\mathrm{eff}}^{\prime} & =n_{\mathrm{eff}}-\frac{1}{2} n_{\mathrm{eff}}^{3} \varepsilon\left[p_{12}-\mu\left(p_{11}+p_{12}\right)\right], \\
\Lambda^{\prime} & =\Lambda[1+(1-P)] \varepsilon,
\end{aligned}
$$

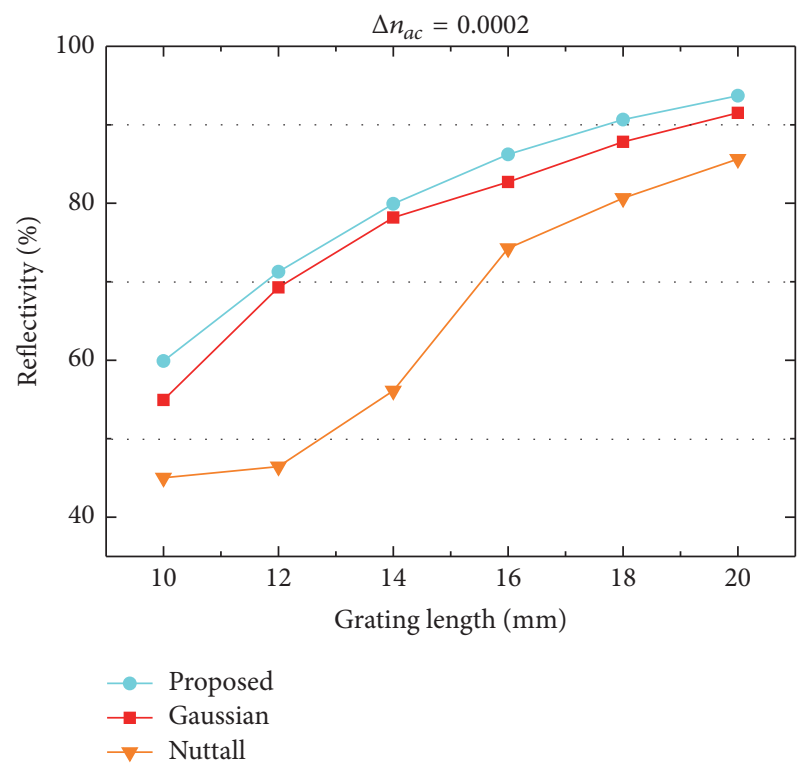

FIGURE 8: Reflectivity versus grating length.

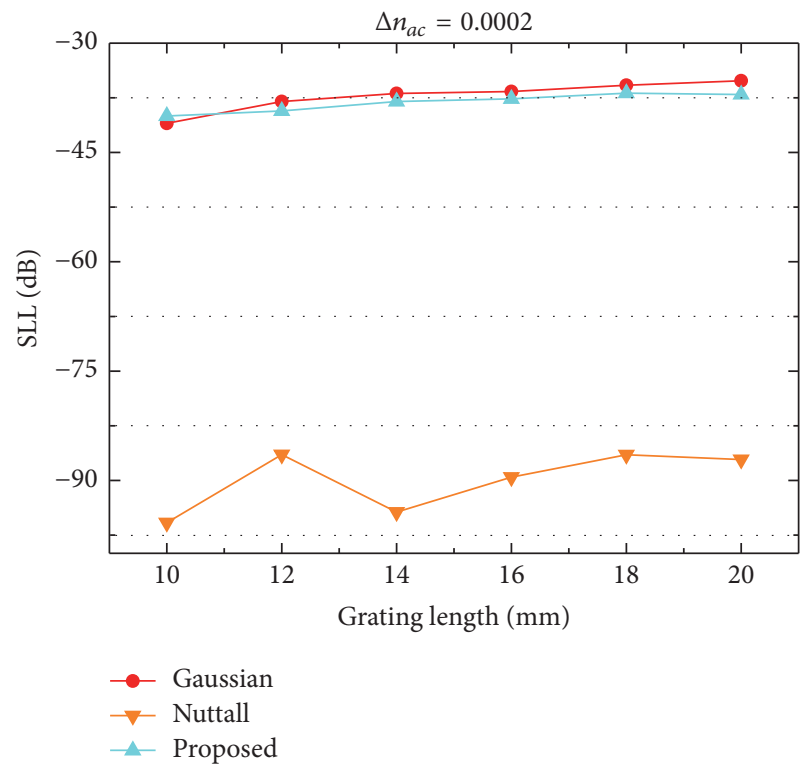

FIGURE 9: Side lobe level versus grating length.

where $n_{\text {eff }}^{\prime}$ is the modified refractive index, $\Lambda^{\prime}$ is the modified grating period, $\varepsilon$ is the axial strain along the fiber, and $P$ is an effective strain optic coefficient [7].

The values of Bragg wavelength shifts using the optimized FBGS are given in Table 1. The applied strain versus Bragg wavelength shift graph is plotted in Figure 11. Fine linear response is seen among the applied strain and Bragg wavelength shift through all the calculated regions, which proves the efficiency of the optimized FBGS.

Nonlinearity effects on strain sensitivity of the proposed apodization are illustrated in Figure 12. The variations of reflectivity have been calculated under the effect of strain 
TABLE 1: Bragg wavelength shift versus the applied strain.

\begin{tabular}{lcccccccccccc}
\hline Microstrain $(\mu \varepsilon)$ & 50 & 100 & 150 & 200 & 250 & 300 & 350 & 400 & 450 & 500 & 550 & 600 \\
\hline Wavelength shift $(\mathrm{nm})$ & 0.064 & 0.128 & 0.192 & 0.256 & 0.321 & 0.385 & 0.443 & 0.497 & 0.561 & 0.625 & 0.689 & 0.741 \\
\hline
\end{tabular}

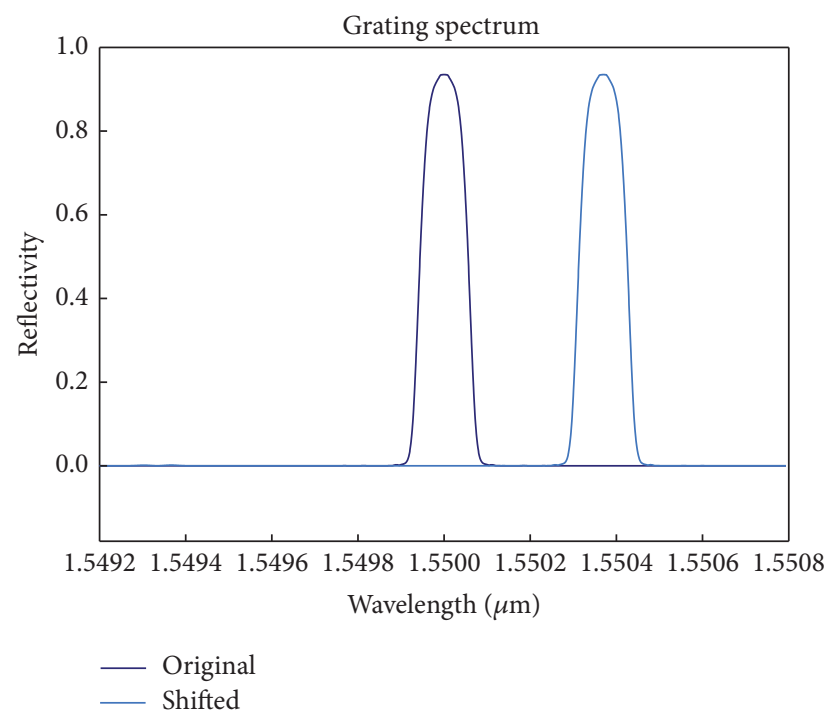

FIgURE 10: Wavelength shifts due to $300 \mu \varepsilon$ applied strain.

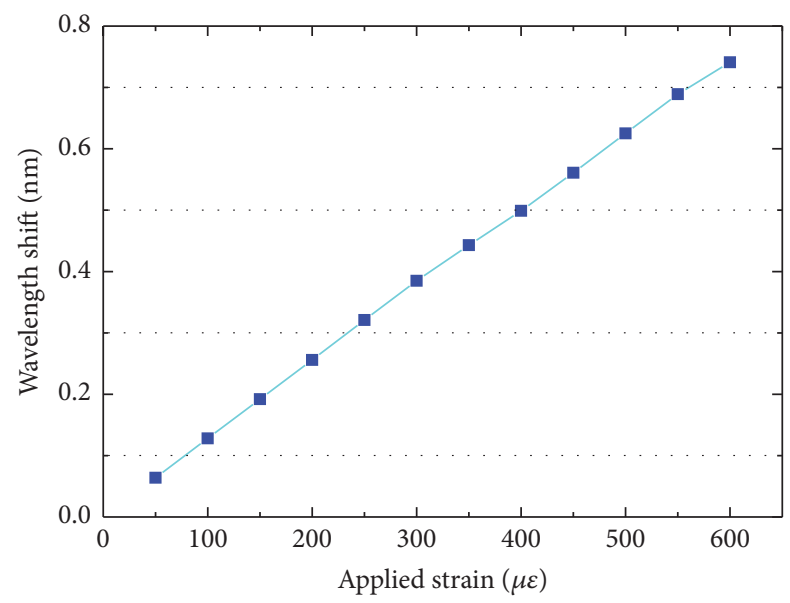

FIGURE 11: Wavelength shifts versus the applied strain.

variation from 0 to $10 \mathrm{MPa}$. The increase that occurs in reflectivity values due to the nonlinearity of the FBG enhances the performance of the FBG. This nonlinearity is explained by the perturbation of molecules when high power signals pass through the fiber.

It can be seen from Figure 12 that the reflectivity increased when $10 \mathrm{MPa}$ strain has been applied in both cases: nonlinear case and linear case, which can be explained due the increased refractive index of glass under strain. The optimized FBGS is then applied in quasi-distributed sensing system of 8 sensors. The purpose of this procedure is to test the reliability of the proposed method in such complexed sensing application.

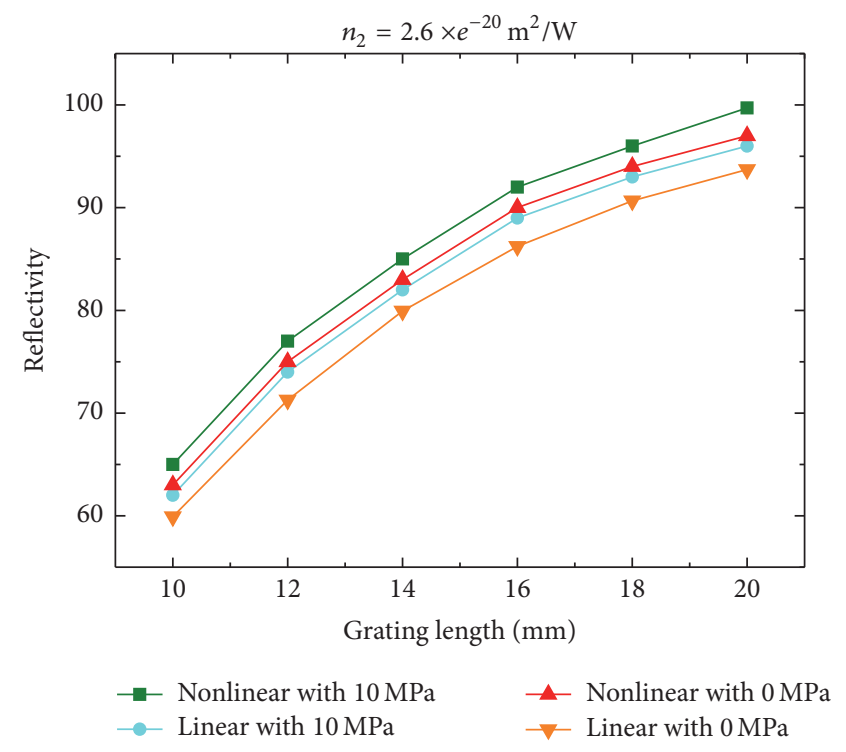

FIGURE 12: Nonlinear effects on strain variation from 0 to $10 \mathrm{MPa}$ with $n_{2}=2.6 \times 10^{-20} \mathrm{~m}^{2} / \mathrm{W}$.

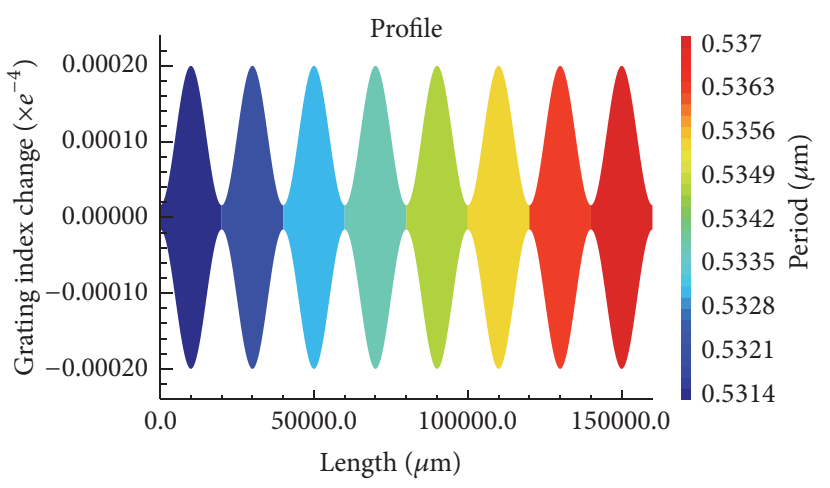

FIGURE 13: The eight hamming apodized FBG strain sensors refractive index profile.

In quasi-distributed sensing systems, side lobes suppression is certainly of much greater importance than in the single sensor case because of the essential need of high adjacent channels isolation to avoid the bad influence that side lobes might have in determining the center frequency of the FBGS. Figure 13 shows the 8 grating refractive index profiles with different colors; each color represents a different grating period $\Lambda$ which has been calculated based on Bragg wavelength $\lambda_{B}$ of each sensor using equation (1).

FBGSs have better resolution with narrower bandwidth, which is more preferable in practice because of the smaller wavelength tunable range that it requires, and then can 


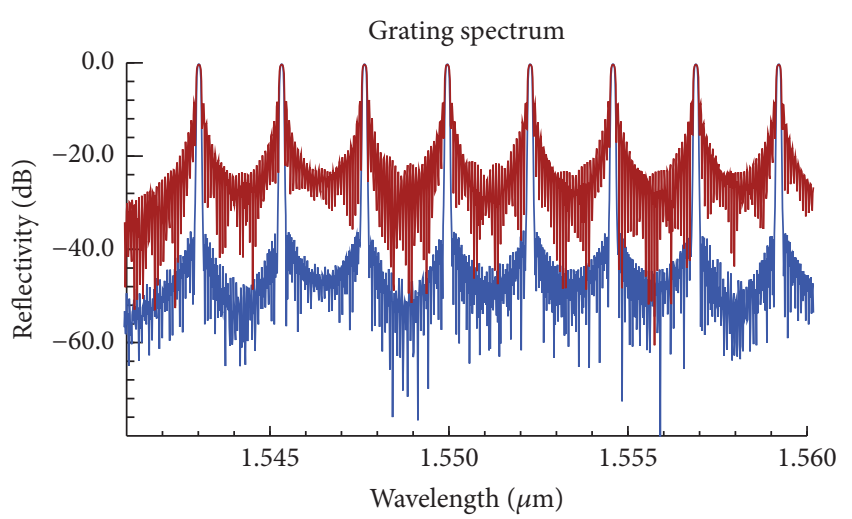

FIGURE 14: The 8 FBG strain sensors with $2 \mathrm{~nm}$ spacing: red: uniform and blue: optimized.

achieve higher measurement speed. Channel spacing of $2 \mathrm{~nm}$ is recommended to be used according to the official ITU-TCband grid [4].

Figure 14 shows the corresponding 8 quasi-distributed FBG-based strain sensors reflection spectrum with $2 \mathrm{~nm}$ channel spacing. The red spectrum is for the 8 Uniform FBG sensors and the blue spectrum is for the 8 optimized FBG sensors. It can be observed that optimized FBGSs provide very good reflectivity, high side lobe suppression (less than $-40 \mathrm{~dB}$ ), and better resolution with narrower bandwidth and then can achieve more accurate measurement and high sensing speed, which proves the efficiency of the proposed apodization optimization.

The 4th optimized FBG is then used for strain measurement with the interest to determine the maximum strain that conserves $0.4 \mathrm{~nm}$ spacing between adjacent channels according to ITU standards. Figure 15(a) illustrates the blue shift on FBG4 reflection spectrum when strain of $1500 \mu \varepsilon$ is applied, which results in complete isolation between FBG4 and FBG5 reflectivity peaks. The red spectrum represents the normal state of the 8 optimized FBGSs.

The maximum allowable strain range that makes FBG4 reflectivity peak at $0.4 \mathrm{~nm}$ from FBG5 reflectivity peak is achieved at strain difference of $1660 \mu \varepsilon$ as shown in Figure 15(b). Remarkable isolation that is higher than $20 \mathrm{~dB}$ is obtained which is enough to make FBG4 (under $1660 \mu \varepsilon$ ) and FBG5 reflectivity peaks distinguishable from each other at the detector receiver.

\section{Conclusion}

In this paper, we introduce novel apodization optimization of FBGS based on reflectivity, FWHM, and SLL to get the most suitable design parameters to be used for strain measurement. The simulation results show that the proposed apodization results in an overall superior performance as it provides higher reflectivity with small FWHM and very low SLL compared with the state-of-art alternatives. As can also be demonstrated, the grating length of $20 \mathrm{~mm}$ and the refractive index of 0.0002 for the apodized FBGS are the most convenient values, respectively. The proposed profile with the optimized parameters has then been used

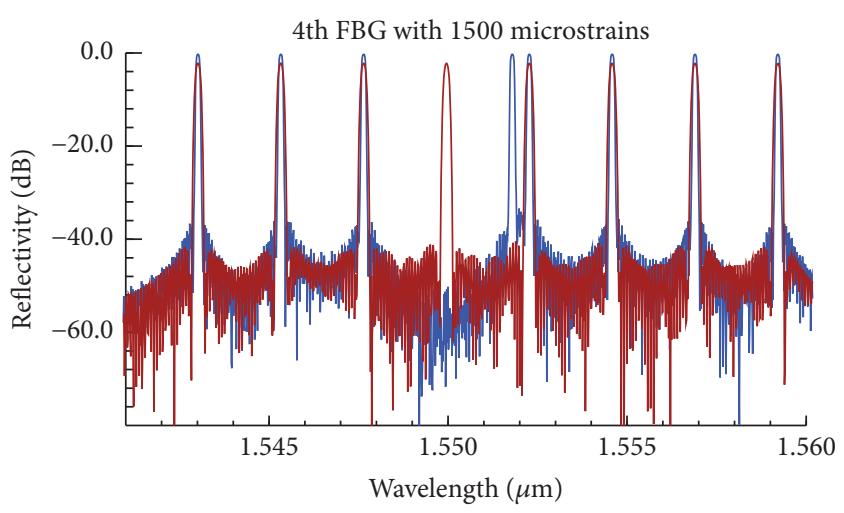

(a)

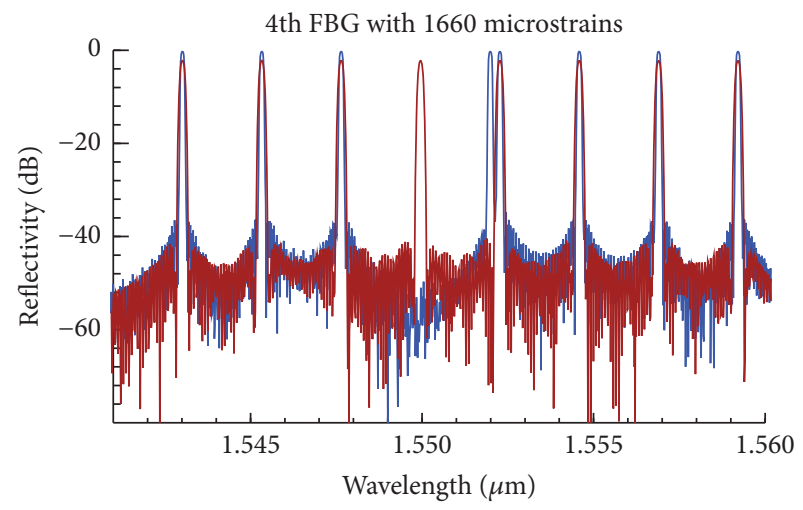

(b)

FIGURE 15: Strain effect on the 4th optimized FBG.

for strain measurement to be evaluated in detection of the applied strain changes. The simulation results prove that the optimized sensor provides very satisfactory performance in detection of the strain changes through the small wavelength shifts in the reflected spectrum. Finally, the optimized FBG strain sensor has been examined in quasi-distributed strain sensing system of 8 sensors to determine the maximum allowable strain range that conserves $0.4 \mathrm{~nm}$ channel spacing between adjacent channels using the proposed method of apodization. Results show that the optimized FBGS offers very good performance and provides the required adjacent channels isolation; therefore channels can be closer to each other due to the negligible effect of side lobes on the adjacent sensors reflectivity peaks. Consequently, the allowable range of strain sensitivity can rise up to $1660 \mu \varepsilon$.

\section{Competing Interests}

The authors declare that there is no conflict of interests regarding the publication of this paper.

\section{References}

[1] T. Geernaert, F. Berghmans, and H. Thienpont, "Microstructured Fibers: butterfly microstructured fiber complements FBG-based sensing," Laser Focus World, pp. 43-47, 2015. 
[2] J. Du, L. Li, X. Fan, Q. Liu, and Z. He, "Sensitivity enhancement for fiber bragg grating sensors by four wave mixing," Photonics, vol. 2, no. 2, pp. 426-439, 2015.

[3] K. S. Khalid, M. Zafrullah, S. M. Bilal, and M. A. Mirza, "Simulation and analysis of Gaussian apodized fiber Bragg grating strain sensor," Journal of Optical Technology, vol. 79, no. 10, pp. 667-673, 2012.

[4] N. A. Mohammed, T. A. Ali, and M. H. Aly, "Evaluation and performance enhancement for accurate FBG temperature sensor measurement with different apodization profiles in single and quasi-distributed DWDM systems," Optics and Lasers in Engineering, vol. 55, pp. 22-34, 2014.

[5] M. J. Moghimi, H. Ghafoori-Fard, and A. Rostami, "Analysis and design of all-optical switching in apodized and chirped Bragg gratings," Progress in Electromagnetics Research B, vol. 8, pp. 87-102, 2008.

[6] M. Chakkour, A. Hajaji, and O. Aghzout, "Design and study of EDFA-WDM optical transmission system using FBG at 10 Gbits/s chromatic despersion compensation effects," in Proceedings of the Mediterranean Conference on Information \& Communication Technologies, Saidia, Morocco, May 2015.

[7] H. Venghaus, Wavelength Filters in Fibre Optics, chapter 5, Springer, Berlin, Germany, 2006.

[8] I. Yulianti, M. Abu Sahmah, M. Sevia, and M. Abdulaziz, "Simulation of apodization profiles performances for unchirped fiber bragg gratings," in Proceedings of the International Conference on Photonics (ICP '10), pp. 4244-7187, Langkawi, Kedah, July 2010. 


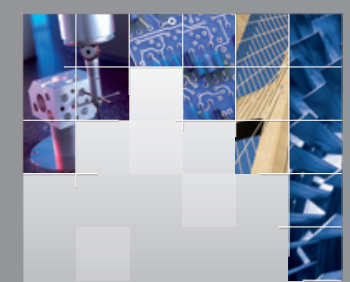

\section{Enfincering}
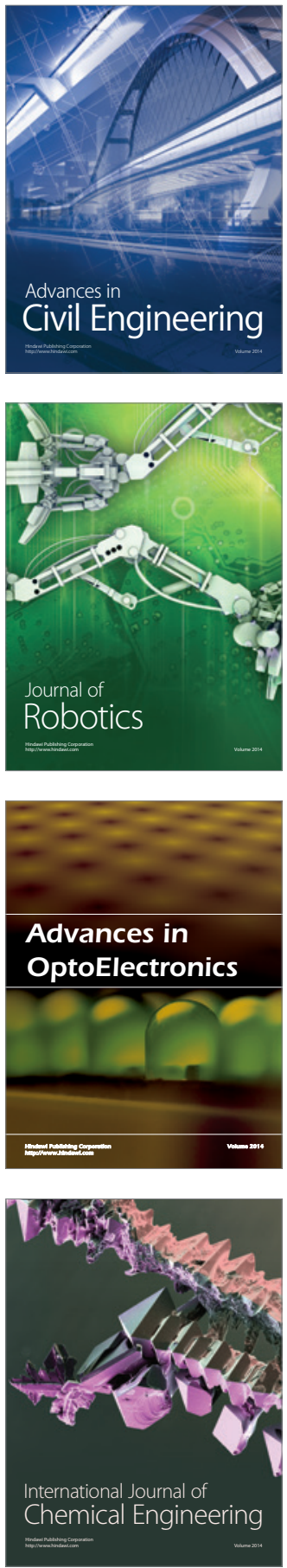

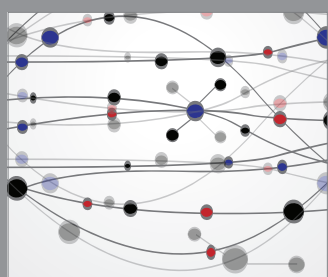

The Scientific World Journal

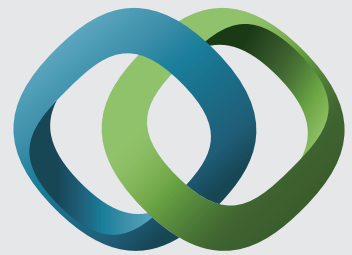

\section{Hindawi}

Submit your manuscripts at

http://www.hindawi.com
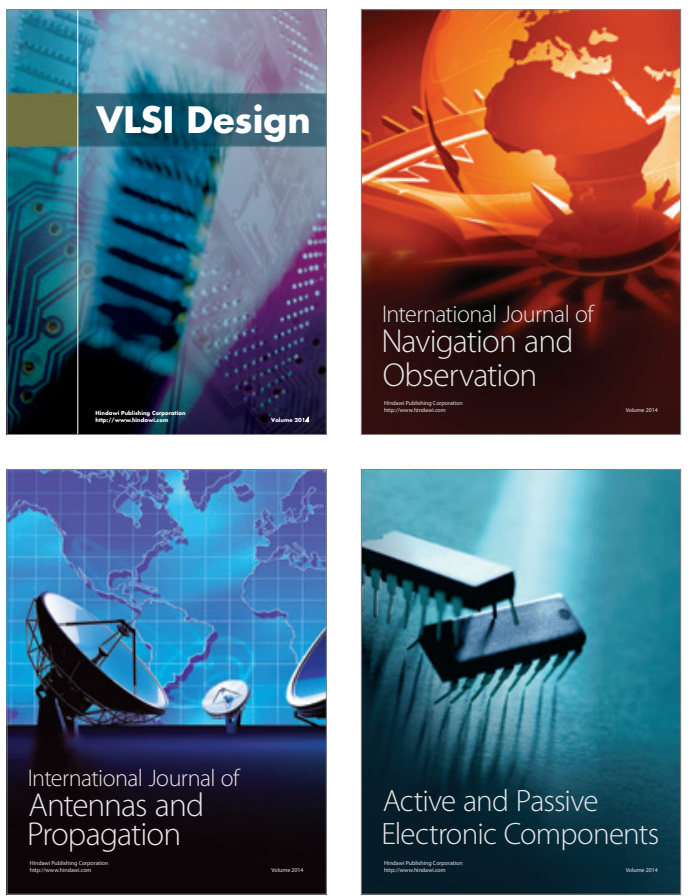
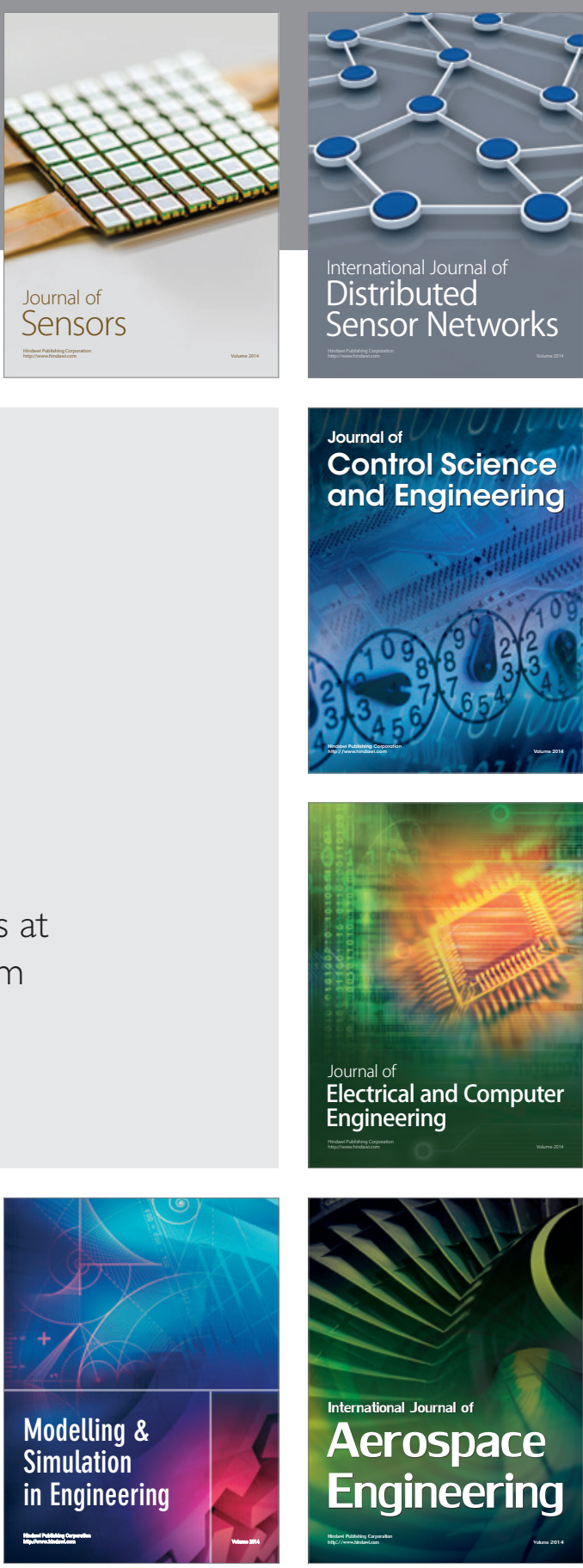

International Journal of

Distributed

Sensor Networks

Journal of

Control Science

and Engineering
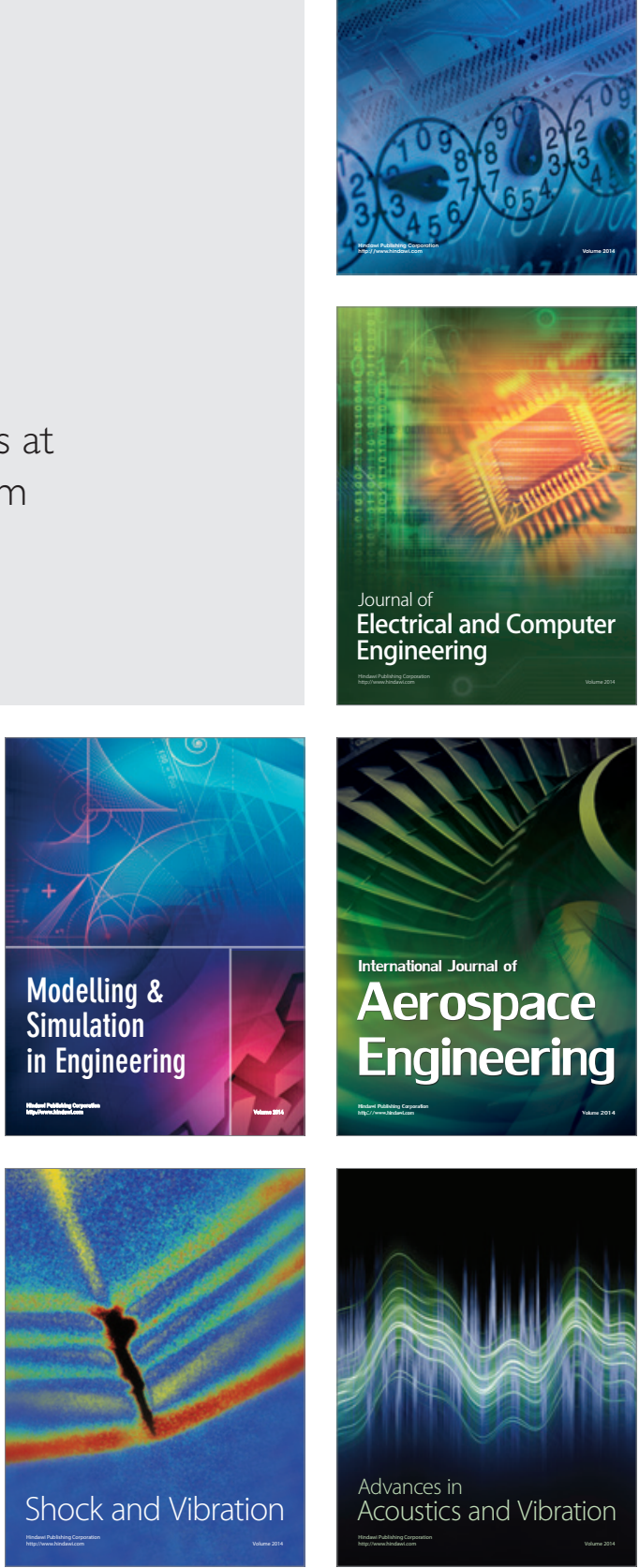